\title{
Coupling of the cell cycle and metabolism in yeast cell-cycle-related oscillations via resource criticality and checkpoint gating
}

\author{
Luke Morgan $^{\mathrm{a}}$, Gregory Moses ${ }^{\mathrm{b}}$ and Todd R. Young ${ }^{\mathrm{a}}$ \\ ${ }^{a}$ Department of Mathematics, Ohio University, Athens, $\mathrm{OH}, \mathrm{USA} ;{ }^{\mathrm{b}}$ Department of Mathematics, University of \\ Cincinnati, Cincinnati, OH, USA
}

\begin{abstract}
We investigate the possibility that slow metabolic, cell-cycle-related oscillations in yeast and associated temporal clustering of cells within the cell cycle could be due to an interplay between near-critical metabolism and cell cycle checkpoints. We construct a dynamical model of the cell cycles of a large culture of cells that incorporates checkpoint gating and metabolic mode switching that are triggered by resource thresholds. We investigate the model analytically and prove that there exist open sets of parameter values for which the model possesses stable periodic solutions that exhibit metabolic oscillations with cell cycle clustering. Simulations of the model give evidence that such solutions exist for large sets of parameter values. This demonstrates that checkpoint gating coupled with critical resources can be a robust mechanism for producing the phenomena observed in experiments.
\end{abstract}

\section{ARTICLE HISTORY}

Received 17 November 2017 Accepted 7 March 2018

\section{KEYWORDS}

Saccharomyces cerevisiae; autonomous oscillations; cell cycle synchrony

\section{Introduction}

Yeast Metabolic Oscillations (YMO) are periodic oscillations of physiological variables in yeast bioreactor experiments that have been studied for over 60 years (see Finn \& Wilson, 1954; Kuenzi \& Fiechter, 1969; von Meyenburg, 1969). There are clearly different types of YMO, and these have been called by different names (Bier, Bakker, \& Westerhoff, 2000; De Monte, d'Ovidio, Danø, \& Sørensen, 2007; Keulers, Satroutdinov, Sazuki, \& Kuriyama, 1996). Several authors classify oscillations as 'glycolytic', 'respiratory' or 'cellcycle-related' (Henson, 2005). All involve stable or quasi-stable periodic oscillations of metabolic variables, such as dissolved oxygen $\left(\mathrm{DO}_{2}\right)$ in the media driven by periodic, culture-wide switching between fermentative and respiratory metabolism modes. Faster oscillations, called glycolytic and respiratory, are independent of the Cell Division Cycle (CDC). This manuscript deals only with cell-cycle-related oscillations and seeks to further the understanding of the how these slower metabolic oscillations and the cell cycle are coordinated. A correlation between slow YMO and the bud index (fraction of cells budded)

CONTACT Todd R. Young youngt@ohio.edu 
was noted as early as (Kuenzi \& Fiechter, 1969; von Meyenburg, 1969), but a link between YMO and the CDC was obscured by the fact that the periods of YMO were shorter than the culture doubling times (a proxy for the CDC period). With the advent of genetic expression data, the correlation between YMO and the CDC was noted again (Henson, 2005; Klevecz \& Murray, 2001; Tu, Kudlicki, Rowicka, \& McKnight, 2005), and the nature of these oscillations has received attention ever since. See Burnetti, Aydin, and Buchler (2016) and its bibliography for a summary of recent research and evidence of coupling between the CDC and YMO.

The relationship between metabolic oscillations and the cell cycle is of basic biological interest because it exposes questions regarding the coordination and interconnectedness of various cellular and genetic processes (Klevecz, 1976; Klevecz \& Murray, 2001; Robertson, Stowers, Boczko, \& Johnson, 2008; Cai \& Tu, 2012). For instance, Zhao, Chen, Carey, and Futcher (2016) and Ewald, Kuehne, Zamboni, and Skotheim (2016) note that the CDC and metabolism have many regulatory genes in common. Additionally, understanding yeast oscillations and regulation of metabolism is important in the efficient management of bioprocesses (Beuse, Kopmann, Diekmann, \& Thoma, 1999; Uchiyama, Morimoto, Yokoyama, \& Shioya, 1996).

Boczko, Stowers, Gedeon, and Young (2010) proposed cell cycle clustering as part of the explanation of the link between YMO and the CDC. They hypothesized that feedback between the metabolism and the CDC could cause groups of cells to segregate into CDC synchronized cohorts. They studied a few simple cell cycle models that included 'blocking' inspired by cell cycle checkpoints. They proposed that a group of cells in the critical S-phase might affect metabolism, and the metabolites may in turn inhibit or promote cell growth in the later part of the G1 phase, thus setting up a coupling mechanism in which YMO oscillation and CDC clustering are inextricably intertwined. They showed analytically and numerically that feedback can robustly cause CDC clustering. By clustering we do not mean spatial clustering (cultures that exhibit YMO occur in well-mixed bioreactors), but temporal clustering - cohorts of cells traversing the CDC in near synchrony (see Figure 1). The mathematical results in Boczko et al. (2010) are similar to those in Chandrasekaran, Achuthan, and Canavier (2011) where, in a different context, it was observed that reciprocal coupling can stabilize a 2-cluster solution by enforcing synchrony within clusters that would not synchronize in isolation.

The mathematical results led to the experimental verification of the occurrence of clustering in two types of oscillating yeast using both bud index and cell density data (Boczko et al., 2010; Stowers, Young, \& Boczko, 2011). Some of the measurements from those experiments are shown in Figure 2. Experiments and analysis in Slavov and Botstein (2011) support this conclusion. In Young, Fernandez, Buckalew, Moses, and Boczko (2012) the authors expanded the analysis to very general forms of feedback and found that cell cycle clustering is a robust phenomenon in dynamical models where cells in one phase produce signals that inhibit growth/progression in another phase. Gong, Moses, Neiman, and Young (2014) studied cell cycle coupling in the presence of random perturbations (noise), such as the dispersive effect of experimentally measured asymmetric division. They concluded that the negative feedback required to allow clusters to form must involve a slow down of at least $30 \%$ relative to normal progression. Such a slow-down can be considered quite 'strong', far from any weak coupling effects. 


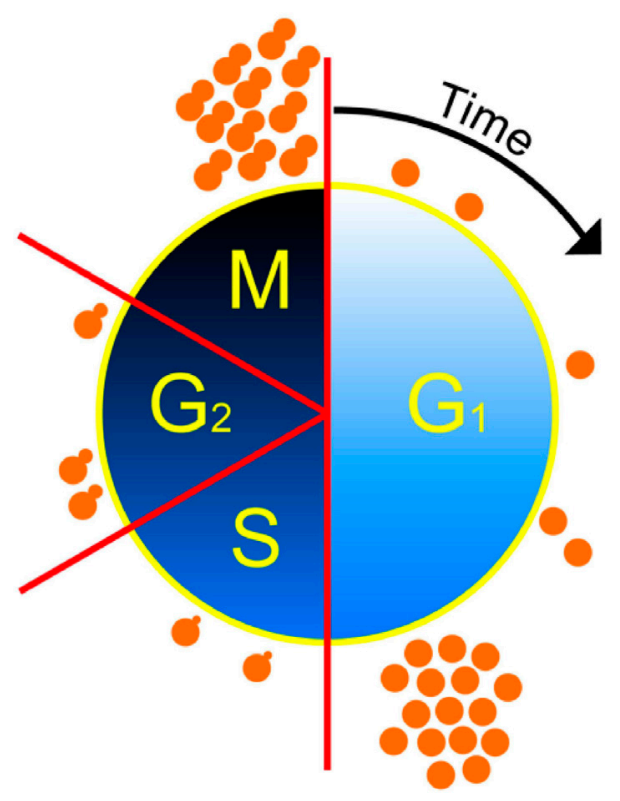

Figure 1. A schematic snapshot of cells in the budding yeast cell cycle with most of the cells forming two cohorts or temporal clusters. These cohorts will progress through the cell cycle in near anti-phase.

Various investigators have studied chemical signalling as the mediator of metabolic synchrony in YMO (Danø, Madsen, \& Sørensen, 2007). For instance, Murray, Klevecz, and Lloyd (2003) proposed that 'endogenously produced acetaldehyde and sulphate tune the oscillation of mitochondrial energization state whereas sulphide mediates population synchrony'. The authors of Henson (2004) and Sohn and Kuriyama (2001) suggest hydrogen sulphide as a synchronizer in respiratory oscillations, while Adams, Kuriyama, Lloyd, and Murray (2003) found that Gts1 protein plays a key stabilizing role. In the context of fast glycolytic oscillations, Danø et al. (2007) found that acetaldehyde has a strong effect on metabolic synchronization (without any cell cycle synchronization), but also that glucose can mediate synchronization if the glucose transporter is not overwhelmed by the amount of glucose. Similarly, some authors (see e.g. Palkova \& Forstova, 2000 and Breeden, 2014) have attributed cell-cycle-related oscillations to various forms of signalling. For instance, Muller, Exler, Aguilera-Vazquez, Guerrero-Martin, and Reuss (2003) suggest a signalling agent, cAMP, that 'plays a major role in mediating the integration of energy metabolism and cell cycle progression, both in the single cell and during cell-cycle-related oscillations in continuous culture, respectively'.

Earlier work focused on the possibility that signalling plays the key role in organizing of cells into clusters in cell-cycle-related oscillations (Young et al., 2012). Here we propose a particular mechanism for signalling that is not via the production of any specific chemical agent or agents but rather through criticality of resources coupled with the engagement of a cell cycle checkpoint. Our hypothesis is an elaboration on the ideas in Martegani, Porro, Ranzi, and Alberghina (1990) where it was proposed that cell cycle 'partial synchronization' in the experiments occurs because fluctuations in ethanol and other metabolites raise and lower thresholds for initiation of budding and division. 


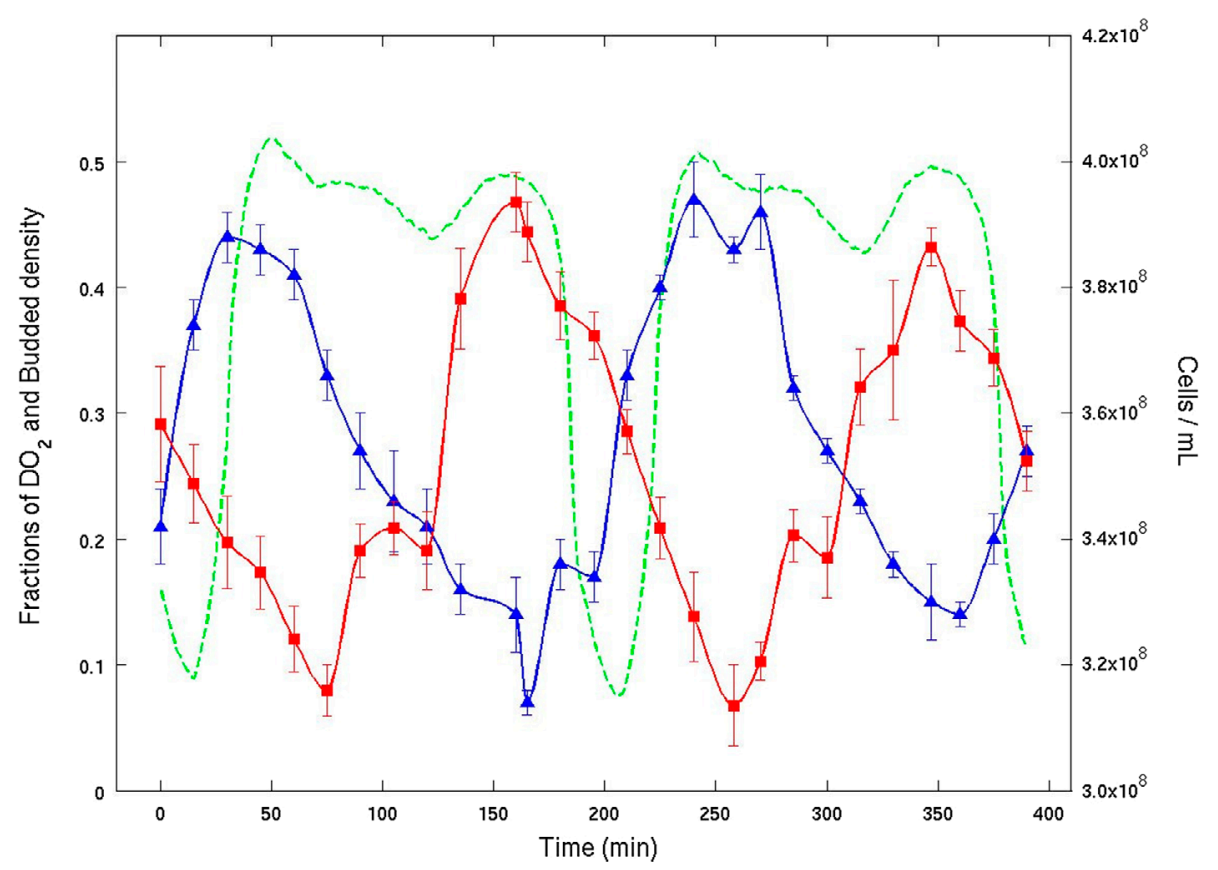

Figure 2. Experimental time series from a continuous culture of budding yeast. Dissolved $\mathrm{O}_{2} \%$ (green dashed), bud index \% (blue with triangles) and cell density (red with squares) are plotted vs. time. The doubling time calculated from the dilution rate in this experiment was $\approx 7 \mathrm{~h}$. The plot shows clearly that the bud index (\% of cells with buds from microscopy) and cell density are both tightly coordinated with the oscillation in dissolved $\mathrm{O}_{2}$. Closer examination shows that the yeast cells are organized into two cohorts traversing the cell cycle. The cells within each cohort are nearly synchronized. One cohort begins budding at about minute 160 and the other cohort begins to bud at about minute 350 . Reproduced from Stowers et al. (2011).

Rotenberg (1977) proposed that periodic gating of cells in a cell cycle could produce a clustered population structure. By periodic gating, we mean that there is a point in the cell cycle that is periodically open or closed to passing cells. We note that periodic engagement of a cell cycle checkpoint could be a mechanism for gating and the relative slow-down produced would be $100 \%$, far stronger than the needed $30 \%$. We propose that checkpoint gating may be crucial in the observed correlation between metabolic oscillations and the cell cycle in YMO experiments. We show that stable periodic- clustered solutions accompanied temporally by metabolic oscillations can be produced in a simple model with a checkpoint that is triggered by near critical resources and differential usage of resources in the cell cycle.

We reason as follows: in YMO experiments, cultures are first grown to high density, i.e. to the carrying capacity of the bioreactor. By definition, resources are critically scarce - they are just sufficient to sustain the population of the culture. Thus, a small fluctuation could push the resources below some critical level. Being below a critical level could cause one of the cell cycle checkpoints, perhaps at G1-S (Start), to stop cells until resources levels recover. Cells that get caught by the checkpoint are then synchronized with each other. Coincidently, availability of resources is also the key determining factor in the mode of metabolism of yeast. Once a cohort (cluster) of synchronized cells forms then this cohort 
could later cause fluctuations in the resources levels, thus causing further checkpoint gating and metabolic switching (between aerobic and anaerobic modes) which reinforces the clustering of cohorts. It is easily conceivable that a stable periodic oscillation would form with most individual cells belonging to synchronized cohorts.

In many papers on yeast metabolic oscillations, it was noted that many different metabolic resources are seen to oscillate (Beuse et al., 1999), such as glycogen and trehalose (Heinzle, Dunn, Furakawa, \& Tanner, 1982). However, they also noted that 'the residual glucose concentration remained nearly constant at a very low level (33-39 mg/l)' pointing to the criticality of this primary resource.

A key assumption in the model we propose is that cells in different phases of the cell cycle may use resources at different rates so that once a cohort of cells has synchronized, it may subsequently cause further fluctuations in metabolites. Differential use of resources in different phases of the cell cycle has been noted in the literature since as early as Kuenzi \& Fiechter (1969). In Munch, Sonnleitner, and Fiechter (1992), it was observed that the cells in the budding phase greatly reduce the consumption of external resources while continuing to consume internal resources. In Duboc, Marison, and von Stockar (1996), it was noted that storage carbohydrates are not consumed during the S-phase and that 'the specific growth rate and biomass production are low during the S-phase and high during the G2, M and G1 phases'. Futcher (2006) noted that slowly growing yeast cells store carbohydrates until the end of the G1 phase at which time they are liquidated and used.

At the same time, coupling of the cell cycle and metabolism was noted in Muller et al. (2003). Cai and Tu (2012) noted changes in metabolism that resulted from cell cycle progression and noted that metabolites may play a key role in the regulation of entry into the S-phase. The paper Newcomb, Diderich, Slattery, and Heideman (2003) found a possible link between the rate of glycolysis and gene expression that is important for the initiation of the S phase. Detailed biochemical models of the growth and division processes within a single fission yeast's cell cycle, including checkpoint controls, were considered in Novak, Csikasz-Nagy, Gyorffy, Chen, and Tyson (1998). Recently, Zhao et al. (2016) and Ewald et al. (2016) observed that the use of internal carbohydrates is regulated by CDK activity. CDKs have long been known to be primary regulatory agents in the cell cycle.

In the next section, we propose a dynamical model that incorporates the following observations: (1) yeast cells switch metabolic modes under critical conditions, (2) metabolic rates are linked to the location in the CDC and (3) the Start checkpoint is triggered metabolically. In Section 3 we analyse the model mathematically and prove that there exist open sets of parameter values for which the model mimics the behaviour observed in cellcycle-related oscillations. In Section 4, we report results from careful numerical simulations of the model. We find that there are large bands in parameter space for which the model produces stable periodic metabolic oscillations accompanied by cell cycle clustering. Our conclusion from these analytical and numerical investigations is that a model incorporating the observations above is adequate to explain the robust clustering of cells into temporal cohorts that has been seen to accompany some metabolic oscillations. 


\section{Model of the cell cycle and metabolism}

We propose and study a dynamical model of the cell division cycle and metabolism for cultures with a large number of cells. We will let the cell cycle of a single cell be represented mathematically as a unit circle, that is, let the state (position in the cell cycle) of the $i$-th cell be denoted $c_{i} \in[0,1) \equiv S^{1}$. We assume that time has been scaled so that all cells move at a rate of 1 around the cycle (unit circle) unless they are being blocked at a checkpoint.

Our model will have a single checkpoint, located in our coordinates at a point $s \in(0,1)$. We will let the variable $z \geq 0$ denote the level of resources. We will suppose that the checkpoint is triggered by a scarcity of resources, that is, there is a checkpoint threshold $\chi$ such that if $z<\chi$ then cells are blocked from proceeding past the checkpoint at $s$.

We assume that cells in one fixed region of the cycle $H \subset(0,1)$ may consume resources at a higher rate. For some of this manuscript, we suppose that $H$ is the union of two intervals, one of which ends at $s, H=[0, s] \cup\left[h_{1}, h_{2}\right]$. We have in mind that the two intervals comprising $H$ may correspond to the G1 and G2 phases of the yeast cell cycle and $s$ is precisely at the G1-S checkpoint.

Suppose that the metabolism of cells in the culture has two different modes, such as the fermentative and respiratory metabolism of yeast, that use resources in different ways. We will suppose that one mode consumes critical resources at a lower rate and another at a higher rate. Let $m$ be a binary variable that denotes the mode of metabolism, $m=0$ denoting the mode with a low rate of metabolism of a critical resource and $m=1$, the mode with a higher rate. Note that $m$ is a variable representing the mode of all the cells in the culture. This is is justified by the sharp swings in metabolic variables observed in experiments. Further, we assume that the mode of metabolism switches as certain thresholds are crossed. Specifically, we assume that $m$ switches from 1 to 0 when $z=\mu$ and from 0 to 1 when $z=v$. We will assume that

$$
0 \leq \mu<\chi<v
$$

Let $a>0$ be the rate at which resources are introduced into the bioreactor minus the rate of metabolism of the culture associated with mode $m=0$. Suppose that while cells are in the region $H$ and in the high metabolism mode they consume resources at a rate that is differentially higher by $b$. Then $z$ is governed by:

$$
\frac{d z}{d t}=a-m b I
$$

where

$$
I(\mathbf{c}) \equiv \frac{\#\left\{i: c_{i} \in H\right\}}{n} \quad \text { (fraction of cells in the region } H \text { ). }
$$

When a cell reaches 1 (division) two cells appear at 0 . However, in this model, the trajectories of the two cells will be identical, so we will only keep track of one of them. In other words, the number of cells is assumed to be fixed. The phase space consists of coordinates $\mathbf{c}=\left(c_{i}\right)$, along with the values of $z \geq 0$ and $m \in\{0,1\}$.

Since cells are indistinguishable in the model, if two cells are initially synchronized or become synchronized in the cell cycle, they will remain so for all time. We will refer to subpopulations of synchronized cells as cohorts, and we may consider particular solutions of the system in which cells are organized into a fixed number of cohorts. For such solutions, 
we may reformulate the model so that cohorts take the place of cells. Let $k$ denote the number of cohorts and let the state of the $j$-th cohort at time $t$ be denoted by $x_{j}(t)$ for $j=1, \ldots, k$. The progress of the $i$-th cohort is given by the equation:

$$
\frac{\mathrm{d} x_{i}}{\mathrm{~d} t}= \begin{cases}0 & \text { if } x_{i}=s \text { and } z<\chi \\ 1 & \text { otherwise. }\end{cases}
$$

The equation governing $z$ remains unchanged when considering cohorts, rather than individual cells. We note that Buckalew (2014) considered a variable $z$ representing the level of a signalling agent and has a mathematical structure that is similar to the model we are considering here.

Proposition 2.1: If $a \neq b$, cells in a solution of the system defined above may not remain at the checkpoint s indefinitely.

Proof: First, we note that if any cell remains at the checkpoint $s$ indefinitely, all cells in the system will synchronize and remain at the checkpoint indefinitely, since there is no mechanism for one cell to pass another, and the cells not at the checkpoint are moving towards the checkpoint at a rate of 1 .

Let all cells be synchronized at $s$ and let $z=z_{0}>\mu$ (by the form of the model). Then either $\dot{z}=a$ or $\dot{z}=a-b I$. Either $a-b(I)=a-b(0)=a$ (if $s \notin H$ ) or $a-b(I)=a-b(1)=a-b$ (if $s \in H$ ). If $\dot{z}=a$ or $\dot{z}=a-b>0$ then either $z_{0}>\chi$ already, or $z$ will reach $\chi$ at time $t=\frac{\chi-z_{0}}{\dot{z}}>0$, at which point blocking will release. If $\dot{z}=a-b<0$ then either $z_{0}>\chi$ already, or $z$ will reach $\mu$ at time $t=\frac{\mu-z_{0}}{a-b}>0$. Once $z=\mu$, metabolism changes to $\dot{z}=a>0$, and blocking will release after time $t=\frac{\chi-\mu}{a}$.

We assume that $a \neq b$ for the remainder of the paper.

The inclusion in the model of a aerobic/anaerobic metabolic switching mechanism is justified by the obvious fact that the metabolism of the culture is observed to switch in the experiments and is consistent with yeast biology. For example, when glucose is plentiful, yeast exhibit culture-wide fermentation and when the glucose drops below a certain point, the culture switches to respiratory metabolism. When the glucose rises again to a certain level the yeast will switch back to fermentation. The switch could be triggered by a threshold as suggested in Burnetti et al. (2016), where evidence supports a mechanism where cells spend time in low oxygen consumptions mode until they reach a metabolic threshold and switch to high oxygen consumptions. In Rolland, Winderick, and Thevelein (2002), various glucose sensing and signalling mechanisms and their genes are described, and it is noted that in the presence of sufficient levels of glucose, S. cervisiae prefer fermentation over respiration. Further, Verduyn, Zomerdijk, van Dijken, and Alexander Scheffers (1984) noted that 'ethanol formation occurred only at initial glucose concentrations exceeding $150 \mathrm{mg} \cdot 1^{-1}$, and the rate of alcoholic fermentation increased with increasing glucose concentrations up to $1000 \mathrm{mg} \cdot 1^{-1}$ glucose'. In Fiechter and Seghezzi (1992), they found that yeast cells respond quickly to changes in external glucose that directly result in increased respiratory functions and leads to ethanol production. They also note that glucose and oxygen are the two main extracellular resources that affect glucose metabolism. 


\section{Existence and local stability of periodic solutions}

\subsection{Cyclic solutions}

The fact that cell-cycle-related oscillations are periodic with periods that are integer divisors of the period of the cell division cycle (Boczko et al., 2010) implies that cohorts in the cell division cycle should remain approximately equidistant (temporally) from each other. Thus biologically realistic solutions to our model should not merely be periodic, but have the stronger property that the timing of the cohorts are 'evenly spaced' in the cell division cycle as defined below in Definition 3.1. The phase space is $\left(S^{1}\right)^{k} \times[0, \infty) \times\{0,1\}$, where $S^{1}$ is the unit circle, and $x \in\left(S^{1}\right)^{k}$ encodes the location of each of $k$ cohorts. The variable $z \in[0, \infty)$ represents the level of resources and $m \in\{0,1\}$ is a discrete variable encoding the metabolic state of the system. Given a point $p$ in the phase space, and taking that point as an initial condition, we may define $p(t)$ for any positive $t$ to be the image of $p$ under the evolution function of the dynamical system at time $t$.

Definition 3.1: $\quad$ Let $p=\left(x_{1}, \ldots, x_{k}, z, m\right)$ be a point in the phase space such that $x_{i}<x_{i+1}$ for $i=1, \ldots, k-1$. Then $p$ is called $k$-cyclic if there exists $T<1$ such that $x_{i}(T)=x_{i+1}(0)$ mod 1 (each cohort moves exactly the state previously occupied by the cohort ahead of it) and furthermore, $z(T)=z$ and $m(T)=m$.

It is convenient to consider $k$-cyclic solutions with initial conditions such that $x_{1}=0$. We remark that $k$-cyclic points lie on periodic orbits, and that the restriction $x_{1}=0$ defines a Poincaré section since $x_{1}(t)$ must return to 0 for some $t>0$. (The derivative of each cell is not always positive, but since cells cannot be blocked by the checkpoint indefinitely in our model, they will eventually return to 0 .)

\subsection{Existence and (local) uniqueness of 2-cyclic solutions}

In related work, where cells were slowed down under the influence of a signalling agent but always move with positive velocity, it was shown that $k$-cyclic solutions existed for any $k$ and any arrangement of parameter values (Young et al., 2012). This cannot be the case here; for example, if the distance between the thresholds is sufficiently large, so that blocking by the checkpoint, when triggered, remains triggered for a complete pass through the cell division cycle, then presumably all cohorts will synchronize, and only the 1-cyclic solution is possible. We prove, however, that other $k$-cyclic solutions can exist for some region of parameter space. We are particularly interested in the 2-cyclic solution - two cohorts having been observed in Boczko et al. (2010) and Stowers et al. (2011).

First we consider the case where $H$ consists of two intervals $H=[0, s] \cup\left[h_{1}, h_{2}\right]$. In the next subsection, we will study the systems when $H$ is a single interval. By parameter space in our model, we mean the choices of $s, h_{1}, h_{2}, \mu, v, \chi, a$, and $b$.

Lemma 3.2: There exists a point in parameter space where a 2-cyclic solution exists and is asymptotically stable. In the orbit of this 2-cyclic solution, every time a cluster reaches the checkpoint it is blocked for some positive time. Specifically, this occurs for $H=[0,0.6] \cup$ $[0.75,0.95]$, metabolic thresholds $\mu=0$ and $v=0.05$, checkpoint threshold $\chi=0.02$ and differential parameters $a=0.15$ and $b=0.4$.

We provide the details of the proof below, but first summarize the method, which is constructive, for this lemma as well as for Corollary 3.3, Lemma 3.4, and Corollary 3.5. We 
observe that the derivatives $\dot{x}_{1}, \dot{x}_{2}, \dot{z}$ are piecewise constant (the first two because cohorts are either moving at unit speed or are being blocked; the latter by the form of $\dot{z}$ and the fact that $I$ takes on a discrete set of values for a fixed number of cells and switches between these values at discrete times). The state variable $m$ is likewise piecewise constant, since $z$ is continuous. We can therefore discretize the trajectory of an initial condition as follows: calculate the maximum time $t_{M}$, such that each derivative (and $m$ ) is constant on $t \in\left[0, t_{M}\right)$, calculate the value of each variable at $t=t_{M}$, and repeat, using these new values as our initial condition. This will produce a piecewise defined solution and a sequence of discrete 'snapshots' of the trajectory at times when the piecewise solution switches forms. By properly selecting our parameters and our initial conditions, we will find that two of these snapshots are identical, that is, the trajectory is a periodic orbit. By asymptotic stability of the solution, we mean asymptotic stability of the periodic orbit traced by the solution. Solutions starting in a small neighbourhood of the orbit will remain in a small neighbourhood of the orbit and converge to the orbit. A small phase shift can occur.

Proof: We prove this theorem by construction. We observe that the dynamics of the system are quite limited; $\dot{z}$ is piecewise constant in time for any initial condition, taking on only a finite number of possible values (in this case, $0.15,-0.25$, and -0.05 ), while the state variable $m$ is also piecewise constant, and the cohorts $x_{i}$ move at one of two rates, 0 or 1. Given an initial condition, it is thus relatively straightforward to chart its trajectory. In particular, we will show via direct computation that $(0,0.57 \overline{3}, 0.01,1)$ is a 2 -cyclic point. When $x_{2}$ reaches $0, x_{1}$ will simultaneously reach $0.57 \overline{3}$; at the same moment, $z$ will again reach 0.01 and $m$ will be 1 .

To begin our construction, we note that the assumption that each cluster experiences blocking every time it reaches 0.6 is sufficient to calculate the initial $z$-value of a 2 -cyclic solution, should one exist, as well as the initial state of $m$. In particular, $x_{2}$ will be held at 0.6 until $z=0.02$, then released. During the time it takes $x_{2}$ to reach 1 , the other cluster $x_{1}$ will remain in $H$ (again, assuming such a solution actually exists). Routine calculations show that $z$ will increase to 0.05 when $x_{2}$ reaches 0.8 , and will then decrease as $x_{2}$ continues to travel towards 1 ; when $x_{2}$ actually reaches $1, z=0.01$ and $m=1$.

From this initial condition, then, the following must happen: $z$ must reach $0, z$ must then reach 0.02 (sometime during which $x_{2}$ must have reached 0.6 ); then, blocking having been released, an additional normalized 0.4 time units must pass for $x_{2}$ to reach 1 . Since we can calculate the time it takes for $z$ to reach 0 from an initial value of 0.01 and the time it then takes to grow to 0.02 , we may calculate the total distance that $x_{1}$, starting from a position at 0 , travels during the time it takes $x_{2}$ to reach 1 : in particular, $0.57 \overline{3}$ normalized units. Thus, if a 2 -cyclic solution exists, it must have the form $(0,0.57 \overline{3}, 0.01,1)$. It is routine to demonstrate that this potential 2-cyclic solution is, in fact, a 2-cyclic solution, and we omit the calculations for reasons of space.

This solution is stable in the $x_{1}$ and $x_{2}$ variables, in an extremely strong sense. Since $x_{2}$ will be held in place at the checkpoint, it is clear that its exact initial position is irrelevant; $x_{2}$ can be perturbed by any amount on the Poincaré section, as long as it is not perturbed so far from the checkpoint that blocking turns off before it reaches it, and that initial condition will not merely converge towards the periodic orbit but merge into it. 
To prove that this solution is stable in the $z$-variable, we simply replace $z$ with $z+\epsilon$ and by brute force compute its value after a single iteration of the Poincaré map. Doing so, we find that in the $z$ variable, stability is again exceptionally strong; after one iteration of the Poincaré map, the perturbed initial condition will return exactly to 0.01 .

Corollary 3.3: The parameter value described in the previous lemma exists in a neighbourhood of such values. That is to say, there exists an open region of parameter space such that asymptotically stable 2-cyclic solutions exist.

Proof: Let the system parameters all be $\epsilon$-close to those of Lemma 3.2. We denote these parameters by e.g. $\hat{a}$ and $\hat{v}$. We note that the dynamics of the system do not depend specifically on the metabolic thresholds $\mu, v$, and $\chi$; rather, it is based on where $z_{0}$ is in relation to the thresholds, and the distance between the thresholds, that is, the time it takes $z$ to cross the gap between them. Therefore, by shifting the system, we may assume that $\mu$ remains 0 .

Following the calculation of Lemma 3.2, we see that if some point exists, its precise location can be calculated. We start by observing that although the system is not continuous, its discontinuities happen predictably at assigned intervals. That is, suppose that all parameters, including the initial value of $z$ and the initial value of $x_{2}$, are assumed to be in a neighbourhood of their values from Lemma 3.2; then the time it takes $x_{2}$ to reach 0.6 , the time it takes for $z$ to drop to 0 and then increase to $\chi$, the time it takes for $z$ to then rise to $v$, the time it takes to then decrease back to 0 , and the time it takes $x_{2}$ to reach 1 , all vary continuously with the system parameters, at least initially. This observation allows us to calculate what $z(0)$ must be, following the calculations of Lemma 3.2 but replacing the values of the lemma with the general parameter values: $z(0)=\hat{v}+(\hat{a}-\hat{b})\left(\hat{h}_{2}-s-\frac{\hat{v}-\hat{x}}{\hat{a}}\right)+(\hat{a}-0.5 \hat{b})\left(1-\hat{h}_{2}\right)$, and $z$ is decreasing, that is $m=1$. We confirm from the equation that the necessary value of $z(0)$ depends continuously on parameters and note that plugging in the parameters of the lemma gives $z(0)=0.01$ as desired.

If $x_{2}$ is close enough to $s$ that it is blocked when it first reaches $s$, then when $x_{2}$ reaches 1 , we have $z=z(0)$ by construction of $z(0)$. We therefore turn our attention to $x_{1}$ and $x_{2}$. It is easy to calculate where $x_{1}$ will fall when $x_{2}$ reaches 1 : it travels at a rate of 1 for $t=\frac{0-z(0)}{a-b}+\frac{\chi}{a}$ (the time it takes for blocking to initiate and release), and since after that time period, $x_{2}$ is at the checkpoint $s$ and moving at a rate of 1 , it then travels an additional $1-s$ units, for a total distance of $1-s+\frac{0-z(0)}{a-b}+\frac{\chi}{a}$. Setting $x_{2}(0)=1-s+\frac{0-z(0)}{a-b}+\frac{\chi}{a}$ will therefore produce our 2-cyclic solution, provided that it is close enough to $s$ that it reaches $s$ before blocking releases; if the parameters are close enough to the parameters of the lemma, than the continuous dependence of parameters in the equation for $x_{2}$ guarantees this.

We have considered stability in the clustered subspace; that is to say that although the oscillators abstract cohorts of large numbers of cells, there are only two oscillators, and stability was checked by perturbing them. If we now view each cohort properly as containing many billions of cells, we can ask a separate but related stability question, which is what happens when the individual cells are slightly perturbed, rather than the entire cohort. The answer is still stability. That is obvious if the cells in $x_{2}$ are perturbed; they will collapse back into a cohort when they are all stopped at the same checkpoint. 
Perturbing $x_{1}$ will slightly affect the evolution of $z$, since cells may be perturbed out of $H$, but a small perturbation of $x_{1}$ will result in a small perturbation of $z$. Thus over the course of an iteration of the Poincaré map, $z$ may be assumed to closely track its non-perturbed value; both cohorts (now loose collections of cells) will therefore experience blocking, which will cause them to collapse back to points; this case is then covered by the corollary.

Fixed points of the Poincaré map, and thus periodic orbits, cannot be assumed to be unique; for example, if $z$ is oscillating 'out of tune', so that the cohorts never experience feedback, there will be an interval of neutral fixed points. However, the stability results above show uniqueness in the biologically interesting region where cohorts are approximately antipodal and blocking occurs every time a cluster reaches a checkpoint.

\subsection{Cyclic solutions with a single $H$ interval}

We consider the existence of 2-cyclic solutions in a system identical to that of Section 3.2 except that the signalling region will be taken solely to be $[0, s]$, without a second region. We motivate our considerations by remarking that the link between the cell cycle and metabolism is not well-understood and so such a model needs to be fairly robust. In the next lemma, we prove the existence of an asymptotically stable periodic orbit (which is not 2-cyclic as in Definition 3.1).

Lemma 3.4: Let $H=[0,0.6], a=0.3, b=1$, and the thresholds be $0<\chi=0.04<v=$ 0.05 then the system has an asymptotically stable periodic orbit with two cohorts.

Proof: The calculations of the previous section, appropriately modified, show that, were a 2 -cyclic solution to exist, then on the Poincaré section, $z=0.035, m=0$, and $x_{2}=\frac{5}{12}$. We will consider the forward trajectory of this point. We first observe that, as $x_{2}$ is somewhat distant from $s=0.6$, blocking will disengage before $x_{2}$ reaches $s$. However, because $z$ falls so rapidly when both the cohorts are in $H$, blocking will re-engage before $x_{2}$ can reach $s$, and $x_{2}$ will in fact be blocked. A routine calculation shows that when blocking releases and $x_{2}$ reaches 1 , the first cluster $x_{1}$ has reached 0.6 and is being blocked $(z=0.035<0.4)$. Thus our point is not, after all, a 2-cyclic solution. However, once blocking releases and $x_{1}$ returns to 1 , the trajectory is back at its initial condition. Stability follows immediately from the form of the model; see the discussion in the proof of Lemma 3.2.

The proof of the following is identical in form to the proof of Corollary 3.3 and is omitted.

Corollary 3.5: For an open region in parameter space, an asymptotically stable periodic orbit, with two cohorts travelling around the unit circle in a nearly antipodal configuration, exists.

\section{Simulations}

We wrote MATLAB programs to complete simulations of the models described in Sections 3.2 and 3.3. We used the programs to search for parameters $a$ and $b$ such that the system exhibits stable 2-cyclic solutions.

Programs simulate a population of one thousand cells evolving under the model for a time equivalent to 30 cell cycles. The $H$ region was fixed for the duration of each simulation. The checkpoint was represented by a small interval $R$. When a solution entered $R$ while 
$z<\chi$ it remained stationary until $z$ became greater than $\chi$ (Equation 2.1). We used an initial value of $z=0.5$ in each simulation. The initial locations of the cohorts $\left(x_{1}=0\right.$ and $x_{2}=0.5$ ) were the same in each run of the program. In each simulation, we applied Euler's method, using a step size of $\frac{3}{1600}$. The step size was determined by $\frac{\text { Cycles }}{2 * \text { Steps }}$. A small white noise perturbation of magnitude 0.0001 was added during all simulations so that only stable solutions would be observed.

In Figure 3(a), we investigated stability in the case where $H=[0,0.65] \cup[0.75,0.9]$ and $R=[0.64,0.65]$. The $H$ regions biologically motivated with $[0, .65]$ being an estimate for the size of the G1 region of the CDC (Boczko et al., 2010). The second $H$ region $[0.75,0.9]$ mimics the $\mathrm{G} 2$ region. We let $\mu=0.1, \chi=0.14$ and $v=0.15$.

In Figure 3(b), we investigated stability in the case where $H=[0,0.85]$ and $R=$ $[0.84,0.85]$. We let $\mu=0.1, \chi=0.125$ and $v=0.15$. We found that relatively small single$H$ regions can produce stable clustered solutions (Lemma 3.4), while letting $H=[0,0.65]$ did not produce stable solutions. Letting $H$ be large $(H=[0,0.85])$ resulted in stable 2clustered solutions. We therefore believe that the single- $H$ region model is more susceptible to changes in parameter values then the two- $H$ region model, which is perhaps more consistent with yeast's G1 and G2 phases.

For both models, we varied the parameter $a$ in $[0.01,0.8]$ and varied $b$ in $[0.01,2.5]$ with grid steps of 0.01 for both parameters. For each combination of $a$ and $b$, we recorded whether the system formed stable 2-cyclic solutions, or, if not, how it failed to do so.

In each simulation (for a fixed pair $(a, b)$ ), our program recorded whether blocking at the checkpoint occurred. We distinguished between a number of cases: either checkpoint blocking never occurred; blocking initially occurred (in the first 4000 time steps), but then settled into an orbit where cohorts did not experience further blocking; or blocking occurred regularly (throughout the entire 8000 time steps). Secondly, we determined whether or not two cohorts had formed at the end of the simulation by checking whether $95 \%$ of the cells were inside two small intervals. When two cohorts appeared, we investigated further to determine whether they had approximately the same number of cells. In each case where two equally sized cohorts appeared, we then determined whether the solution was cyclic. We did this by examining the position of the cohorts for the last 2000 time steps and checking whether the distance between the cohorts stayed within a range we determined to be acceptable. This acceptable range was $\left[0.5-\frac{\text { time of blocking }}{2}-0.01,0.5+\right.$ $\left.\frac{\text { time of blocking }}{2}+0.01\right]$.

Our observations are colour coded in Figure 3: a black dot indicates that either two cohorts never formed, or that blocking never occurred, a red dot indicates that for those values the trajectory settled into a two-cohort periodic solution but that the solution did not experience blocking after the first 4000 steps, yellow dots indicate non-cyclic periodic 2-cluster solutions, while green dots indicate a 2-cyclic solution was observed, and that blocking occurred throughout the running time of the program.

In Figure 3, we see that many parameter values produced 2-cyclic solutions. Each subfigure of Figure 3 is the result of twenty thousand simulations. We observe that for each of the $a$ values $(a>0.15)$, there is a range of $b$ values such that green dots occur. Hence for each of the $a$ values, there is a range where 2-cyclic solutions can occur dependent upon the $b$ value.

We observe that solutions occurred for parameters in 'bands' that alternated between different solution types. For example in Figure 3(a), a vertical band of red solutions was 


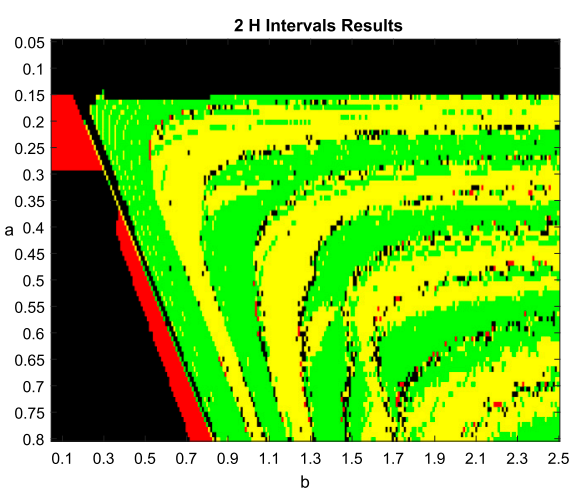

(a)

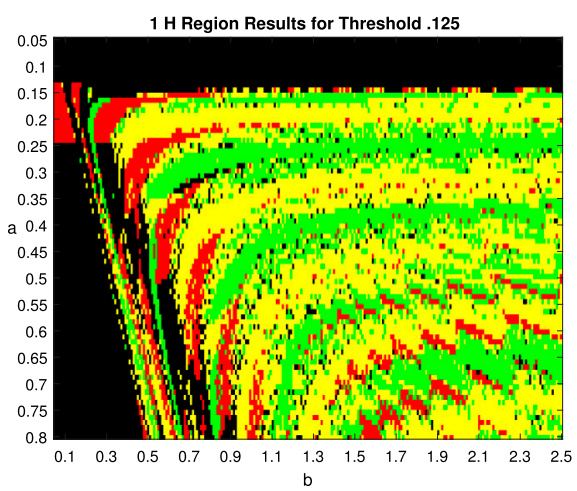

(b)

Figure 3. The results of a parameter search investigating the formation of asymptotically stable 2cyclic solutions as described in Corollary 3.3 (left) and Corollary 3.5 (right). The same initial conditions, $z(0)=0.5, x_{1}(0)=0.0$, and $x_{2}(0)=0.5$, were used in each simulation. We varied the values of $a$ and $b$, simulated thirty cell cycles with a population of one thousand cells and recorded the results. A green dot indicates that the simulation produced a locally asymptotically stable, 2-cyclic solution. The other colours represent different types of failure of the system to exhibit an asymptotically stable, cyclic, 2-cluster solution. A black dot indicates that either two cohorts never formed, or that blocking never occurred, a red dot indicates that for those values the trajectory settled into a two-cohort periodic solution but that the solution did not experience blocking after the first 4000 steps, yellow dots indicate non-cyclic periodic 2-cluster solutions. In frame (a), $H=[0,0.65] \cup[0.75,0.9], R=[0.64,0.65]$, $\mu=0.1, \chi=0.14$ and $v=0.15$. In frame (b), $H=[0,0.85], R=[0.84,0.85], \mu=0.1, \chi=0.125$ and $v=0.15$.

produced which was followed by alternating green and yellow bands. The mechanism behind this pattern requires further investigation. We note that growing the culture to carrying capacity in the experiments corresponds to moving the parameters in the simulations. Thus parameters can be expected to cross through these bands of stable periodic solutions as the experiment proceeds to its steady state and will presumably have opportunity to become 'locked' in one of the green bands.

We include only two representative figures, but remark that similar figures result for other choices of parameter values. We further note that in other experiments, we considered initial conditions where the cells were not perfectly clustered, but were within intervals of length 0.05 centred around 0.025 and 0.525 ; this had no effect on the end state of the system, thus further demonstrating the asymptotic stability of the solution (see our discussion after the proof of Corollary 3.3).

\section{Conclusions}

Although types of YMO are complex and varied, it is known that in at least some cases, they are accompanied by clustering in the cell division cycle (Boczko et al., 2010; Stowers et al., 2011). It is further known that the mechanism of checkpoints periodically engaging and disengaging in the cell division cycle can lead to such clustering (Rotenberg, 1977). However, a self-driven periodic gating is not motivated by the biology of the system rather, since the Start checkpoint is known to be linked to resource availability, one might better expect that the checkpoint will engage and disengage in response to this, while the 
consumption of resources is known to be tied with the location of cells in the CDC (Duboc et al., 1996; Futcher, 2006).

Guided by these facts, we have investigated a feedback system where the location of cells in the CDC influences the rise and fall of metabolic agents; these in turn control the checkpoints of the system, which control the location of the cells, resulting in two-way coupling. The relationship between metabolism and the CDC is not sufficiently wellunderstood to be modelled with high precision, but we have proposed and studied a toy model as proof of concept. We find that the combination of three uncontroversial facts - that yeast cells metabolize at different rates under starvation conditions, that metabolic rates are linked with the CDC, and that the Start checkpoint is triggered metabolically can result in a locally stable solution (Corollary 3.3) corresponding to cell-cycle-related YMO. Guided by these analytical results, we find in simulation that the formation of locally stable cohorts is a robust phenomenon, occurring for a wide range of parameter values (Section 4). Regions of stability appear as bands in parameter space that must be crossed as parameters are varied, such as must occur in the experiments as the cultures are grown to carrying capacity. These bands give the system opportunity to 'lock' into a stable solution as the capacity is approached. We have further shown that these results are robust against changes in the model (Section 3.3); even such basic properties as connectedness of the high-metabolism region are not essential to the model.

We infer from this study that the occurrence of cell-cycle-related YMO could potentially be explained by an interplay between near-critical metabolism and cell cycle checkpoints. This hypothesis could be test experimentally by attempting to detect indicators of engagement of the checkpoint for short periods just before the observed budding events, e.g. at about 150 and $350 \mathrm{~min}$ in Figure 2. Clues in this direction are suggested in the studies Klevecz and Murray (2001) and Cai and Tu (2012), but we could not find direct evidence in those publications.

\section{Disclosure statement}

No potential conflict of interest was reported by the authors.

\section{Funding}

This work was supported by Division of Mathematical Sciences [grant number 1418787].

\section{References}

Adams, C. A., Kuriyama, H., Lloyd, D., \& Murray, D. B. (2003). The Gts1 protein stabilizes the autonomous oscillator in yeast. Yeast, 20(6), 463-470. doi:10.1002/yea.976

Beuse, M., Kopmann, A., Diekmann, H., \& Thoma, M. (1999). Oxygen, pH Value, and carbon source induced changes of the mode of oscillation in synchronous continuous culture of Saccharomyces cerevisiae Biotechnology and Bio. Engineering, 63(4), 410-417.

Bier, M., Bakker, B. M., \& Westerhoff, H. V. (2000). How yeast cells synchronize their glycolytic oscillations: A perturbation analytic treatment. Biophysical Journal, 78, 1087-1093.

Boczko, E. M., Stowers, C. C., Gedeon, T., \& Young, T. R. (2010). ODE, RDE and SDE models of cell cycle dynamics and clustering in yeast. Journal of Biological Dynamics, 4, 328-345.

Breeden, L. L. (2014). $\alpha$-Factor synchronization of budding yeast. Methods Enzymology, 283, 332 342. 
Buckalew, R. (2014). Cell cycle clustering and quorum sensing in a reponse/signaling mediated feedback model. DCDS B, 19(4), 867-881.

Burnetti, A. J., Aydin, M., \& Buchler, N. E. (2016). Cell cycle Start is coupled to entry into the yeast metabolic cycle across diverse strains and growth rates. $M B o C, 27,64-74$.

Cai, L., \& Tu, B. P. (2012). Driving the Cell Cycle Through Metabolism. Annual Review of Cell and Developmental Biology, 28, 59-87.

Chandrasekaran, L., Achuthan, S., \& Canavier, C. C. (2011). Stability of two cluster solutions in pulse coupled networks of neural oscillators. Journal of Computational Neuroscience, 30, 427-445.

Danø, S., Madsen, M. F., \& Sørensen, P. G. (2007). Quantitative characterization of cell synchronization in yeast. PNAS, 104, 12732-12736.

De Monte, S., d'Ovidio, F., Danø, S., \& Sørensen, P. G. (2007). Dynamical quorum sensing: Population density encoded in cellular dynamics. PNAS, 104, 18377-18381.

Duboc, P., Marison, L., \& von Stockar, U. (1996). Physiology of Saccharomyces cerevisiae during cell cycle oscillations. Journal of Biotechnology, 51, 57-72.

Ewald, J. C., Kuehne, A., Zamboni, N., \& Skotheim, J. M. (2016). The yeast cyclin-dependent kinase routes carbon fluxes to fuel cell cycle progression. Molecular Cell, 62(4), 532-45. doi:10.1016/j.molcel.2016.02.017.

Fiechter, A., \& Seghezzi, W. (1992). Regulation of glucsoe metabolism in growing yeast cells. Journal of Biotechnology, 27, 27-45.

Finn, R. K., \& Wilson, R. E. (1954). Population dynamic behavior of the Chemostat system. Agricultural and Food Chemistry, 2, 66-69.

Futcher, B. (2006). Metabolic cycle, cell cycle and the finishing kick to start. Genome Biology, 7, $107-111$.

Gong, X., Moses, G., Neiman, A., \& Young, T. R. (2014). Noise-induced dispersion and breakup of clusters in cell cycle dynamics. The Journal of Theoretical Biology, 335, 160-169.

Heinzle, E., Dunn, I. J., Furakawa, K., \& Tanner, R. D. (1982). Modeling of sustained oscillations observed in continuous culture of Saccharomyces Cerevisiae, Modeling and control of biotechnical processes 1st IFAC. Workshop, 1982, 57-65.

Henson, M. A. (2004). Modeling the sychronization of yeast respiratory oscillations. Journal of Theoretical Biology, 231, 443-458.

Henson, M. A. (2005). Cell ensemble modeling of metabolic oscillations in continuous yeast cultures. Computers \& Chemical Engineering, 29, 645-661.

Keulers, M., Satroutdinov, A. D., Sazuki, T., \& Kuriyama, H. (1996). Synchronization affector of autonomous short period sustained oscillation of Saccharomyces cerevisiae. Yeast, 12, 673-682.

Klevecz, R. R. (1976). Quantized generation time in mammalian cells as an expression of the cellular clock. PNAS, 73, 4012-4016.

Klevecz, R. R., \& Murray, D. (2001). Genome wide oscillations in expression. Molecular Biology Reports, 28, 73-82.

Kuenzi, M. T., \& Fiechter, A. (1969). Changes in carbohydrate composition and trehalose activity during the budding cycle of Saccharomyces cerevisiae. Archives of Microbiology, 64, 396-407.

Martegani, E., Porro, D., Ranzi, B. M., \& Alberghina, L. (1990). Involvement of a cell size control mechanism in the induction and maintenance of oscillations in continuous cultures of budding yeast. Biotechnology and Bioengineering, 36(5), 453-459.

Muller, D., Exler, S., Aguilera-Vazquez, L., Guerrero-Martin, E., \& Reuss, M. (2003). Cyclic AMP mediates the cell cycle dynamics of energy metabolism in Saccharomyces cervisiae. Yeast, 20, 351-367.

Munch, T., Sonnleitner, B., \& Fiechter, A. (1992). The decisive role of the Saccharomyces cervisiae cell cycle behavior for dynamic growth characterization. Journal of Biotechnology, 22, 329-352.

Murray, D., Klevecz, R., \& Lloyd, D. (2003). Generation and maintenance of synchrony in Saccharomyces cerevisiae continuous culture. Experimental Cell Research, 287, 10-15.

Newcomb, L. L., Diderich, J. A., Slattery, M. G., \& Heideman, W. (2003). Glucose regulation of Saccharomyces cerevisiae cell cycle genes. Eukaryotic Cell, 2, 143-149. 
Novak, B., Csikasz-Nagy, A., Gyorffy, B., Chen, K., \& Tyson, J. J. (1998). Mathematical model of the fission yeast cell cycle with checkpoint controls at the G1/S, G2/M and metaphase/anaphase transitions. Biophysical Chemistry, 72, 185-200.

Palkova, Z., \& Forstova, J. (2000). Yeast colonies synchronize their growth and development. Journal of Cell Science, 113, 1923-1928.

Robertson, J. B., Stowers, C. C., Boczko, E. M., \& Johnson, C. H. (2008). Real-time luminescence monitoring of cell-cycle and respiratory oscillations in yeast. PNAS, 105, 17988-93.

Rolland, F., Winderick, J., \& Thevelein, J. M. (2002). Glucose-sensing and -signalling mechanisms in yeast. FEMS Yeast Research, 2, 183-201.

Rotenberg, M. (1977). Selective synchrony of cells of differing cycle times. Journal of Theoretical Biology, 66, 389-398.

Slavov, N., \& Botstein, D. (2011). Coupling among growth rate response, metabolic cycle, and cell division cycle in yeast. Molecular Biology of the Cell, 22, 1999-2009.

Sohn, H. Y., \& Kuriyama, H. (2001). Ultradian metabolic oscillation of Saccharomyces cerevisiae during aerobic continuous culture: Hydrogen sulphide, a population synchronizer, is produced by sulphite reductase. Yeast, 18(2), 125-135.

Stowers, C. C., Young, T. R., \& Boczko, E. M. (2011). The structure of populations of budding yeast in response to feedback. Hypotheses in the Life Sciences., 1, 71-84.

Tu, B. P., Kudlicki, A., Rowicka, M., \& McKnight, S. L. (2005). Logic of the yeast metabolic cycle: Temporal compartmentation of cellular processes. Science, 310, 1152-1158.

Uchiyama, K., Morimoto, M., Yokoyama, Y., \& Shioya, S. (1996). Cell cycle dependency of rice $\alpha$-amylase production in a recombinant yeast. Biotechnology and Bioengineering, 54, 262-271.

Verduyn, C., Zomerdijk, T. P. L., van Dijken, J. P., \& Alexander Scheffers, W. (1984). Continuous measurement of ethanol production by aerobic yeast suspensions with an enzyme electrode. Applied Microbiology and Biotechnology, 19, 181-185.

von Meyenburg, H. K. (1969). Energetics of the Budding Cycle of Saccharomyces cerevisiae during Glucose Limited Aerobic Growth. Archives of Microbiology, 66, 289-303.

Young, T., Fernandez, B., Buckalew, R., Moses, G., \& Boczko, E. (2012). Clustering in Cell Cycle Dynamics with General Responsive/Signaling Feedback. Journal of Theoretical Biology, 292, 103115.

Zhao, G., Chen, Y., Carey, L., \& Futcher, B. (2016). Cyclin-dependent kinase co-ordinates carbohydrate metabolism and cell cycle in S. cerevisiae. Molecular Cell, 62, 546-557. 